\title{
PhyloPrimer: a taxon-specific oligonucleotide design platform
}

\author{
Gilda Varliero ${ }^{\text {Corresp., } 1}{ }^{,}$Jared Wray $^{1}{ }$, Cédric Malandain ${ }^{2}$, Gary Barker ${ }^{1}$ \\ ${ }^{1}$ School of Life Sciences, University of Bristol, Bristol, UK \\ 2 Environmental Expertise, HYDREKA, Lyon, France \\ Corresponding Author: Gilda Varliero \\ Email address: gilda.varliero@bristol.ac.uk
}

Many environmental and biomedical biomonitoring and detection studies aim to explore the presence of specific organisms or gene functionalities in microbiome samples. In such cases, when the study hypotheses can be answered with the exploration of a small number of genes, a targeted PCR-approach is appropriate. However, due to the complexity of environmental microbial communities, the design of specific primers is challenging and can lead to non-specific results. We designed PhyloPrimer, the first user-friendly platform to semi-automate the design of taxon-specific oligos (i.e. PCR primers) for a gene of interest. The main strength of PhyloPrimer is the ability to retrieve and align GenBank gene sequences matching the user's input, and to explore their relationships through an online dynamic tree. PhyloPrimer then designs oligos specific to the gene sequences selected from the tree and uses the tree non-selected sequences to look for and maximize oligo differences between targeted and non-targeted sequences, therefore increasing oligo taxon-specificity (positive/negative consensus approach). Designed oligos are then checked for the presence of secondary structure with the nearest-neighbor (NN) calculation and the presence of off-target matches with in silico PCR tests, also processing oligos with degenerate bases. Whilst the main function of PhyloPrimer is the design of taxon-specific oligos (down to the species level), the software can also be used for designing oligos to target a gene without any taxonomic specificity, for designing oligos from preselected sequences and for checking predesigned oligos. We validated the pipeline on four commercially available microbial mock communities using PhyloPrimer to design genus- and species-specific primers for the detection of Streptococcus species in the mock communities. The software performed well on these mock microbial communities and can be found at https://www.cerealsdb.uk.net/cerealgenomics/phyloprimer. 


\section{PhyloPrimer: a taxon-specific oligonucleotide design platform} 2

3 Gilda Varliero ${ }^{1}$, Jared Wray ${ }^{1}$, Cédric Malandain ${ }^{2}$, Gary Barker ${ }^{1}$ 4

$5 \quad{ }^{1}$ School of Life Sciences, University of Bristol, Bristol, UK

$7 \quad{ }^{2}$ Environmental expertise, HYDREKA, Lyon, France

11 Corresponding Author:

12

18 Email address: gilda.varliero@bristol.ac.uk 
19

20

21

22

23

24

25

26

27

28

29

30

31

32

33

34

35

36

37

38

39

40

41

42

43

44

45

46

47

48

49

50

51

52

53

54

55

56

\section{Abstract}

Many environmental and biomedical biomonitoring and detection studies aim to explore the presence of specific organisms or gene functionalities in microbiome samples. In such cases, when the study hypotheses can be answered with the exploration of a small number of genes, a targeted PCR-approach is appropriate. However, due to the complexity of environmental microbial communities, the design of specific primers is challenging and can lead to non-specific results. We designed PhyloPrimer, the first user-friendly platform to semi-automate the design of taxon-specific oligos (i.e. PCR primers) for a gene of interest. The main strength of PhyloPrimer is the ability to retrieve and align GenBank gene sequences matching the user's input, and to explore their relationships through an online dynamic tree. PhyloPrimer then designs oligos specific to the gene sequences selected from the tree and uses the tree non-selected sequences to look for and maximize oligo differences between targeted and non-targeted sequences, therefore increasing oligo taxon-specificity (positive/negative consensus approach). Designed oligos are then checked for the presence of secondary structure with the nearest-neighbor $(\mathrm{NN})$ calculation and the presence of off-target matches with in silico PCR tests, also processing oligos with degenerate bases. Whilst the main function of PhyloPrimer is the design of taxon-specific oligos (down to the species level), the software can also be used for designing oligos to target a gene without any taxonomic specificity, for designing oligos from preselected sequences and for checking predesigned oligos. We validated the pipeline on four commercially available microbial mock communities using PhyloPrimer to design genus- and species-specific primers for the detection of Streptococcus species in the mock communities. The software performed well on these mock microbial communities and can be found at https://www.cerealsdb.uk.net/cerealgenomics/phyloprimer.

\section{Introduction}

The Polymerase Chain Reaction (PCR) is a pivotal technique to many molecular protocols and is widely used to exponentially amplify a specific portion of DNA (e.g. gene) using DNA or RNA template (e.g. the entire DNA or RNA content of an environmental sample), primers, deoxynucleotides (dNTPs), DNA polymerase and reaction buffers (Garibyan \& Avashia, 2013). Before starting with any PCR-based procedure, primers need to be selected to target the specific DNA region and organisms. The amplification starts where the primers anneal to the DNA template, for this reason the specificity of the PCR reaction is highly impacted by the specificity of the primers to the DNA template.

The design of new oligonucleotides (i.e. primers or probes), hereafter abbreviated as oligos, is a relatively easy task when working with known axenic cultures or known low complexity communities but can be challenging when dealing with unknown organisms and complex environment communities. Different studies can require different level of oligo-specificity: 
57 oligos could be designed to target the same DNA portion in all the community organisms (e.g.

58

59

60

61

62

63

64

65

66

67

68

69

70

71

72

73

74

75

76

77

78

79

80

81

82

83

84

85

86

87

88

89

90

91

92

93

94

95

96 universal primers), in a specific group of organisms or in a specific species or strain. The latter two tasks become challenging when the target DNA fragment is present in non-target organisms that are part of the community (Fierer et al., 2005).

Many different primer and probe sequences have been published. These oligos can target a broad variety of different DNA sequences and can present a wide range of target organism's specificity. Universal oligos, such as primers targeting housekeeping genes (e.g. 16S rRNA gene) are widely used for the study of microbial diversity and in diagnostic surveys (e.g. Takahashi et al., 2014). It is also possible to target non-universal genes, such as the nifH gene (e.g. Gaby and Buckley, 2012) and the $p m o A$ gene (e.g. Wang et al., 2017), in order to target only organisms with a specific metabolism and that occupy specific environmental niches. Oligos can also have a more specific target: they can amplify only genes present in organisms of interest even when the gene is present in a wider selection of organisms (e.g. You and Kim, 2020; Yu et al., 2005). When no predesigned oligos are available, however, it is necessary to develop new ones. Oligo sensitivity is a trade-off between the specificity of the oligo to the DNA template and allowing some oligotemplate mismatch if targeting different organisms in order to get an even coverage of all the representative organisms (Parada, Needham \& Fuhrman, 2016). Depending on the user needs, there are many web-tools and software packages freely available for the oligo design. Some of the most widely used tools for primer design are Primer3 and its web interface Primer3Plus (Untergasser et al., 2007, 2012), Oligo7 (Rychlik, 2007) and Primeclade (Gadberry et al., 2005). To target unknown genes where only the protein or related gene sequences are known, it is necessary to design degenerate oligos. The latter take advantage of the codon degeneracy property of the amino acid sequences and, having degenerate bases in their sequences, represent a pool of unique primers that target the same amino acid coding sequence. Primer design tools for degenerate primers can require the input of proteins, such as CODEHOP (Rose, Henikoff \& Henikoff, 2003; Boyce, Chilana \& Rose, 2009) or Primer Premier (Singh et al., 1998); or the input of DNA sequences or alignments, such as DegePrimer (Hugerth et al., 2014), HYDEN (Linhart \& Shamir, 2005) or FAS-DPD (Iserte et al., 2013).

Environmental communities pose many challenges for the oligo specificity as we often do not know what organisms are present and therefore it is difficult to foresee the possible nonspecific products (Morales \& Holben, 2009; Deiner et al., 2017). In silico PCR is an essential step towards the design of specific oligos (Yu \& Zhang, 2011). Some of the commonly used tools are UCSC In-Silico PCR (Kent et al., 2002), FastPCR (Kalendar, Lee \& Schulman, 2009) and Primer-BLAST (Untergasser et al., 2012). The latter allows to one check the oligo specificity against the comprehensive NCBI databases (Sayers et al., 2020). Further to their taxonomic specificity, oligos need to be tested for different parameters, such as the absence of homopolymer regions or di-nucleotide repetitions and the presence of a GC clamp (Elbrecht, Hebert \& Steinke, 2018). Primers must also be scanned for the presence of secondary structures

Peer) reviewing PDF | (2020:10:54347:1:1:NEW 8 Feb 2021) 
97 such as self-dimers, cross-dimers and hairpins (Chuang, Cheng \& Yang, 2013). The analysis of

98 secondary structure $\Delta \mathrm{G}$ is integrated in the pipeline of widely used oligo design software, such as

99 Oligo 7 (Rychlik, 2007) and Primer3 (Untergasser et al., 2012), or can be performed with

100 specific software, such as PrimerROC (Johnston et al., 2019). The characteristics of the targeted

101 organisms must also be taken in consideration. For instance, prokaryotic genomes rarely have

102 introns as gene splicing is rare in these organisms (Sorek \& Cossart, 2010), whereas introns and

103 multiple splicing sites are widely present in eukaryotic genomes and must be taken in

104 consideration when designing primers (Goel, Singh \& Aseri, 2013; Shafee \& Lowe, 2018).

105

106 In case the PCR target is a gene possessed only by a specific organism, the primers can be

107 designed directly on that gene sequence. If more than one gene variant needs to be amplified

108 (e.g. multiple species are targeted), a consensus sequence can be calculated and the oligos can

109 then be designed on it (consensus primers). A consensus sequence is created from a sequence

110 alignment and is defined as a sequence that reports the most frequent base present in the

111 alignment in each position. The construction of this sequence, and consequently the designed

112 oligos, is greatly influenced by the selection of the initial sequences. This pivotal step is usually

113 not implemented in the oligo design software as these require the upload of preselected

114 sequences. To date, only ARB implemented a toolkit that allows the creation of new primers and

115 probes on sequences selected from the ARB phylogenetic tree of ribosomal sequences (Ludwig

116 et al., 2004; Essinger et al., 2015). However, in order to work on other DNA portions, the user

117 needs to create a sequence database to import inside the software.

118 Other tools, such as Morphocatcher (Shirshikov et al., 2019) and Uniqprimer (Karim et al.,

119 2019), propose high specificity primers. This is achieved by comparing the sequences that are the

120 target of the PCR amplification with non-target sequences. However, no help in the sequence

121 selection through phylogenetic tree visualization is available. Therefore in most tools, prior to

122 the oligo design, the user has to retrieve the sequences of interest from a database (e.g. NCBI

123 database), making sure that the sequences represent the DNA portion of interest and that they

124 cover the same sequence fragment. This process can be complex and time-consuming especially

125 when working with environmental microbial communities or working with a ubiquitous and

126 divergent gene.

127

128 We present PhyloPrimer, a user-friendly and comprehensive online platform to i) select the DNA 129 sequences to use for oligo design, ii) construct a consensus sequence, iii) design microbial oligos

130 (i.e. primers), iv) test for oligo specificity through in silico tests and v) test the oligos for the

131 presence of secondary structures with the nearest-neighbour (NN) model for nucleic acids. In

132 addition it provides a unique platform to check oligos (i.e. primer pairs, primer and probe assays,

133 and single oligos) for both secondary structure and non-specific targets. The real strength of

134 PhyloPrimer is the DNA sequence selection where the user can explore the diversity of the

135 sequence of interest through a dynamic phylogenetic tree. The sequences used for the tree

136 construction are retrieved from a modified version of the GenBank database (Sayers et al., 2019) 
137 and are used by the software to increase taxon-specificity (down to the species level) of the

138 designed oligos (positive/negative consensus approach). PhyloPrimer can design both primers

139 and probes for PCR and qPCR applications, however, no qPCR-related tests were performed.

140

141 To test the efficiency and usability of PhyloPrimer we used the $r p o B$ gene, which is a universal

142 gene and encoding the $\beta$-subunit of RNA polymerase (Adékambi, Drancourt \& Raoult, 2009).

143 This is an essential enzyme to all the transcription processes in a cell as it accounts for the

144 synthesis of mRNA, tRNA and rRNA. Its sequence is less conserved across different genomes

145 compared to the 16S rRNA gene. This makes it less suitable to design universal primers but

146 more suitable to design primers that can target specific organisms (Case et al., 2007). We tested

147 PhyloPrimer by designing PCR primers suitable to the detection of organisms belonging to the

148 Streptococcus genus and specific Streptococcus species (Streptococcus agalactiae,

149 Streptococcus pneumoniae, Streptococcus pyogenes, Streptococcus mutans and Streptococcus

150 mitis), amplifying taxon-specific rpoB genes from known mock communities.

\section{$151 \quad 2$ Materials and methods}

\section{$152 \quad 2.1 \quad$ Implementation}

153 PhyloPrimer runs on a remote server provided from the University of Bristol. The current server has 48 CPUs (64-bit Intel(R) Xeon(R) CPU E5-2680 v3 at 2.50GHz). Only 4 PhyloPrimer processes at one time are allowed on the server, the excess processes enter a queue. On average, the oligo design requires 40-50 minutes whereas the oligo check requires 5-10 minutes. The web interface was implemented in HTML and JavaScript. PhyloPrimer is coded in Perl, JavaScript, HTML, CSS and MySQL. Two JavaScript packages were used: a modified version of PhyloCanvas v 1.7.3 (http://phylocanvas.org) and CanvasJS v 2.3.2 (https://canvasjs.com). The user can access PhyloPrimer through a web platform at https://www.cerealsdb.uk.net/cerealgenomics/phyloprimer. PhyloPrimer was tested and implemented using the Safari, Firefox, Chrome browsers. The website uses General Data Protection Regulation (GDPR) cookie acceptance box on the first use. All the PhyloPrimer scripts are also available through the PhyloPrimer GitHub page (https://github.com/gvMicroarctic/PhyloPrimer).

\subsubsection{General workflow and Dynamic Selection}

The PhyloPrimer web platform is structured with sequential web pages that can be categorized into four different groups: i) the home page, ii) the input pages, iii) the oligo pages and iv) the result page. From the home page, the user can select one of the three different input pages available for uploading the data (e.g. DNA sequences, DNA alignments and Newick trees) where each page corresponds to a different modality to use PhyloPrimer. Once the data are uploaded,

172 the user is redirected to the oligo pages where there are different parameter settings to design

173 either primer assays, primer and probe assays or single oligos. Once the user submits these 
174 parameters, the oligo design and the oligo check are performed on the web server. As soon as

175 PhyloPrimer has finished the analyses, the user receives an email with a link to the result page

176 where the user can explore the designed oligos and choose the ones which will be used for future

177 work (Fig. 1).

178

179 PhyloPrimer can be used in three different modalities. It can be used to design oligos from DNA 180 sequences interactively selected from a dynamic phylogenetic tree (Dynamic Selection; Fig. 2A), 181 to design oligos from preselected DNA sequences (Premade Selection; Fig. 2B) and to check 182 predesigned oligos (Oligo Check; Fig. 2C). The Dynamic Selection modality is the strength of

183 PhyloPrimer and was developed to facilitate the selection and retrieval of NCBI sequences for

184 the oligo design. The processes reported in the rest of the manuscript describe this modality and

185 details on the others can be found in the manual at

186 https://github.com/gvMicroarctic/PhyloPrimer.

187

The user can upload one or more sequences representing the same DNA portion (e.g. same gene or gene fragment belonging to different organisms). PhyloPrimer then runs MegaBLAST (Morgulis et al., 2008; Baxevanis, 2020) against the database DB1 (details in Section 2.4). The user can set up three BLAST parameters: the e-value (the probability of finding a match by chance), the identity percentage (the percentage of bases shared between the query and the subject sequence), and coverage percentage (the percentage of bases of the query sequence that are covered by the subject sequence). If more than four matching sequences were found in the database, PhyloPrimer runs a MAFFT alignment (Katoh \& Standley, 2013) and then constructs a phylogenetic tree with FastTree (Price, Dehal \& Arkin, 2009). The user can explore the dynamic tree and look at the sequence information connected to each retrieved GenBank entry (e.g. taxonomy). The user can then select, in the tree, the sequences that must be used for the consensus calculation and therefore the oligo calculation (Fig. 3A).

\section{0}

\subsubsection{Consensus calculation and primer specificity}

201

202

PhyloPrimer uses a consensus approach for the oligo design or, in other words, it designs the

203 oligos from a consensus sequence. After the user selects the adequate sequences from the dynamic tree, PhyloPrimer calculates two consensus sequences. The positive consensus is the consensus calculated from the selected sequences and the one used for the oligo design. The negative consensus is calculated from the sequences that were not selected from the tree and is used to increase the oligo specificity to the target organisms looking at the base difference between the two consensuses (Fig. 3B). After the consensus construction, Phyloprimer compares the two sequences and finds the differing positions. To create taxon-specific oligos, PhyloPrimer uses this information when scoring the oligos with the aim to retrieve the best ones to be visualized in the dynamic result page (Fig. 3C). To guarantee a high level of oligo-specificity, PhyloPrimer also runs an in silico BLAST search where oligos that are specific for the targeted organisms are selected. The user can specify if the oligos must be species-, genus-, family-, 
213 order-, class-, phylum- or domain-specific. PhyloPrimer picks which are the organisms of

214 interest from the phylogenetic tree selections.

\section{2.1.3 Oligo design and scoring system}

216 The consensus sequence can be uploaded to PhyloPrimer by the user through the Premade

217 Selection page or it can be calculated by PhyloPrimer itself. The software constructs the

218 consensus with the DNA sequences or alignments uploaded through the Premade Selection page

219 or with the sequences that were selected by the user on the dynamic tree (Dynamic Selection

220 mode). In order for PhyloPrimer to find suitable oligos, the consensus must have one or more

221 conserved regions, DNA regions that are in common among all the selected/uploaded sequences.

222 If no conserved regions are present, the consensus sequence will be represented by long stretches

223 of degenerate bases and the software will not be able to design any oligo from it. There can be

224 different reasons for this: i) the sequence selection was too broad for the target gene family, ii)

225 the selected sequences did not include only sequences from the same gene family, iii) the

226 sequences represented different DNA regions of the same gene or iv) the studied gene family is

227 very divergent. In general, it is more likely to have a conserved region in the consensus when

228 working with closely related sequences, for example, when developing oligos for a specific

229 species rather than for an entire gene family. However, when the aim is to develop oligos at gene

230 level, the presence of a conserved gene region between different organisms highly depends on

231 the gene sequence. It is essential to know the gene family object of the study and to check the

232 consensus sequence that PhyloPrimer reports. In case the consensus presents a lot of degeneracy,

233 it will be necessary to adjust the maximum number of degenerate bases allowed inside the oligo

234 sequence in the oligo design pages. If this does not help, the design of different oligos for

235 different cluster of organisms should be considered.

236

237

In PhyloPrimer the conserved region of the consensus sequence is determined by the maximum

238

239 number of degenerate bases that is allowed inside the oligo sequences. For instance, if the user sets the maximum degenerate base value to 1 , PhyloPrimer will discard all the oligos that have more than 1 degenerate base in the sequence or, in other words, won't consider the areas of the consensus that have an incidence of degenerate bases higher than 1 base every oligo length (between 18-22 bp by default).

245

PhyloPrimer will start the oligo design only once the positive consensus has been obtained. For each possible oligo length, the software extracts from the consensus sequence all the possible subsequences of that length (Fig. 4A). This first step creates the starting pool of oligos that the following steps will check and discard if not respecting all the design parameters. The first check step discards by default the oligos that are not unique in the consensus sequence, that have homopolymer repetition longer than 3 bases, dinucleotide repetition longer than 6 bases, a GC content lower than $40 \%$ or higher than $60 \%$, and will check and discard the oligos that do not have between 2 and $4 \mathrm{Gs} / \mathrm{Cs}$ in the last 5 bases of 3' oligo end (GC clamp). PhyloPrimer will also check if the oligos have a higher number of degenerate bases than the limit and that only the 
253

254

255

256

257

258

259

260

261

262

263

264

265

266

267

268

269

270

271

272

273

274

275

276

277

278

279

280

281

282

283

284

285

286

287

288

289

290

291

292

correct degenerate bases are present (all except from $\mathrm{N}$ by default). The default number of degenerate bases is set by PhyloPrimer in relation to how many degenerate bases were found inside the consensus sequence but can be changed by the user (Fig. 4B).

PhyloPrimer then calculates the reverse complement of all the oligos and considers the original oligos as putative forward primers and the oligo reverse complements as putative reverse primers (Fig. 4C). All the forward and reverse primers are progressively checked to have a valid melting temperature (between $54{ }^{\circ} \mathrm{C}$ and $64{ }^{\circ} \mathrm{C}$ by default) and, in case the presence of degenerate bases is allowed, not to have degenerate bases in the last 5 bases of the $5^{\prime}$ oligo end and last 2 bases of the 3 ' end oligo tails (by default). The software also checks for the presence of self-dimer and hairpin secondary structures and discards any oligos with a secondary structure associated to a $\Delta \mathrm{G}$ value lower than -5 and $-3 \mathrm{kcal} \mathrm{mol}^{-1}$, respectively (Fig. 4D). Primers meeting the above criteria are then considered as suitable primer pairs (Fig. 4E). The primer pairs are first selected considering the distance between their 5 ' ends on the consensus (between 200 and 600 bases by default). All the suitable primer pairs are then checked for the presence of cross-dimer formations and discarded if the $\Delta \mathrm{G}$ values are lower than $-5 \mathrm{kcal} \mathrm{mol}^{-1}$ (by default). Furthermore the primer pairs are also discarded if the melting temperature difference between forward and reverse primers is higher than $5{ }^{\circ} \mathrm{C}$ or the annealing temperature does not range between $50^{\circ} \mathrm{C}$ and $60{ }^{\circ} \mathrm{C}$ (Fig. 4F).

At this point, all the remaining primer pairs have all the requirements that were set by the user through the oligo pages. All the following steps aim to retrieve the best primer pairs that will be visualized in the result page. This is achieved by assigning points to each primer pair as follows: 1 point is assigned to the primer pair if the melting temperature of the forward and reverse primers differ by less than $1{ }^{\circ} \mathrm{C}$, for each secondary structure 1 point is assigned if the $\Delta \mathrm{G}$ value is higher than $-1 \mathrm{kcal} \mathrm{mol}^{-1}$. Moreover, 20 points are assigned if a base polymorphic between the positive and the negative consensus is present in the last base of the $3^{\prime}$ end and 10 points if it is present in the penultimate base. Two points are also assigned for each additional base difference between the positive and the negative consensus (Fig. 4G). The best 1000 primer pairs are selected and checked for cross-dimer formation (Fig. 4H).

PhyloPrimer selects the first 500 primer pairs that scored the highest points according to the scoring system (Fig. 4I). The oligos belonging to those first 500 primer pairs are BLAST searched against DB2 (details in Section 2.4). PhyloPrimer then screens the BLAST results and retrieves the database sequences that matched both to the forward and reverse primers and uses them to perform a global alignment with Bowtie (Langmead, 2010). PhyloPrimer then checks the alignment results and considers only the database sequences that were matched by both the forward and the reverse primers of a primer pairs. If that sequence belongs to one of the species that were selected from the dynamic tree, PhyloPrimer assigns 10 points to the primer pair, if the species was not among the selected species it deducts 40 points, and every time there is a new correct species PhyloPrimer adds 20 points to the total. By default, PhyloPrimer does not assign more points to primers that belong to the same genus (or higher ranks) of the selected tree 
293

294

295

296

297

298

299

300

301

302

303

304

305

306

307

308

309

310

311

312

313

314

315

316

317

318

319

320

321

322

323

324

325

326

327

328

329

330

331

332

entries. But if these visualization parameters are checked, PhyloPrimer will assign 20 points to the entries that belong to the same taxonomy and deduct 40 to those that do not. This is for facilitating the design of oligos that are specific for a specific genus (or higher taxonomic group) rather than only specific to certain species. In case an additional file was uploaded by the user for an additional BLAST check, PhyloPrimer will also BLAST all the oligos against that database but the outcome will not be the object of the scoring system (Fig. 4J).

The described scoring criteria are all active by default but any of those can be deselected by the user on the Oligo Design page. PhyloPrimer then selects the first 100 primer pairs and these primer pairs will be the ones showed in the last Result Page. When degenerate bases are present inside the oligo sequences, the melting temperature and the GC content are calculated as the mean of these values in each of the possible oligo (Fig. 4L).

The design process for primer pair/probe assays and single oligos is very similar to that described above and is described fully in the software manual.

\subsubsection{Melting temperature and $\Delta G$ secondary structures}

PhyloPrimer calculates oligo melting temperatures $\left(\mathrm{T}_{\mathrm{m}}\right)$ and secondary structure Gibbs free energies $(\Delta \mathrm{G})$ with the nearest-neighbor $(\mathrm{NN})$ model for nucleic acids. This model predicts the thermodynamic behavior of a DNA molecule using the thermodynamic parameters of each nucleotide pair composing the molecule itself. Both the $\mathrm{T}_{\mathrm{m}}$ and the $\Delta \mathrm{G}$ calculation rely on the use of the thermodynamics parameters enthalpy $(\Delta \mathrm{H})$ and entropy $(\Delta \mathrm{S})$. These parameters were derived from calorimetry and spectroscopic experiments of DNA duplexes for nucleotide base pair motives (SantaLucia \& Hicks, 2004), internal mismatches (Allawi \& Santalucia, 1997; Allawi \& SantaLucia, 1998a,b,c; Peyret et al., 1999), dangling ends (Bommarito, Peyret \& SantaLucia, 2000) and hairpin terminal mismatches (unpublished data). The latter were retrieved from the UNAFold database (Markham \& Zuker, 2008). The $\Delta \mathrm{H}$ and $\Delta \mathrm{S}$ are considered temperature independent when working with nucleic acids and are reported for $1 \mathrm{M} \mathrm{Na}^{+}$ conditions.

The melting temperature $\left(\mathrm{T}_{\mathrm{m}}\right)$ of a DNA molecule is the temperature in which half of the DNA is paired with its complement and half is single-stranded. The correct calculation of this parameter is essential to the correct calculation of the PCR annealing temperature, and it is pivotal for the qPCR probe when wanting to differentiate amplicon expression levels.

PhyloPrimer calculates $\mathrm{T}_{\mathrm{m}}$ with the formula reported in SantaLucia \& Hicks (2004). The annealing temperature, $T_{a}$, is calculated as the lowest melting temperature (if more than one oligo is present) minus 5 . This is an indicative calculation as the optimal annealing temperature can considerably vary in relation to the polymerase that is used during the PCR.

The $\Delta \mathrm{G}$, or Gibbs free energy, estimates if a reaction can occur spontaneously ( $\Delta \mathrm{G}$ higher than 0 , exergonic reaction) or not ( $\Delta \mathrm{G}$ lower than 0 , endergonic reaction) and therefore indicates how 
333 stable a particular DNA structure is at a certain temperature. In this case, $\Delta \mathrm{G}$ represents the

334 quantity of energy needed to fully break a secondary structure. The lower it is (more negative),

335 the more stable and likely to occur the secondary structure will be and the more energy will be

336 required to break it. $\Delta \mathrm{G}$ is defined as equal to the enthalpy minus the product of the temperature

337 times the entropy (Gibbs free energy equation). PhyloPrimer calculates the $\Delta \mathrm{G}$ for three different

338 secondary structure formations: self-dimers (i.e. dimers formed within the oligo itself), cross-

339 dimers (i.e. dimers formed between different oligos) and hairpin loops (i.e. hairpin-like

340 secondary structures formed within the oligo itself). For each of these different structures,

341 different rules must be applied to $\Delta \mathrm{H}$ and $\Delta \mathrm{S}$ calculation which are then used to apply Gibbs free

342 energy equation (SantaLucia \& Hicks, 2004).

343

344

Melting temperature and $\Delta \mathrm{G}$ values obtained in this way (SantaLucia \& Hicks, 2004; Gibbs free energy equation) are valid only in $1 \mathrm{M} \mathrm{Na}^{+}$condition. Because the PCR conditions can span a wide range of different conditions, salt correction formulas must be applied to correct the obtained values (Owczarzy et al., 2004, 2008). Depending on the polymerase used and the PCR protocol, $\mathrm{Mg}^{2+}$ and monovalent ions can vary considerably and rarely the $1 \mathrm{M} \mathrm{Na}^{+}$condition is respected. Therefore, PhyloPrimer performs salt-correction correction with the parameters reported and customized in the oligo pages therefore calibrating the corrections on the user specific PCR conditions.

352

When dealing with degenerate oligos, PhyloPrimer calculates melting temperature and $\Delta \mathrm{G}$ values for all the possible oligos. The final $\mathrm{T}_{\mathrm{m}}$ is the average of all the calculated $\mathrm{T}_{\mathrm{m}}$ whereas the final $\Delta \mathrm{G}$ is the lowest $\Delta \mathrm{G}$. More information on the $\mathrm{T}_{\mathrm{m}}$ calculation, $\Delta \mathrm{G}$ calculation and correction formulas, together with all the thermodynamic parameters, can be found in the manual.

\subsubsection{Databases}

PhyloPrimer uses external nucleotide sequence databases in two points of the pipeline. The first point is when it BLAST searches the sequences uploaded in the Dynamic Selection mode to retrieve similar sequences and construct a dynamic phylogenetic tree (DB1), and the second when it checks the oligo specificity through in silico PCR (DB2). DB1 is constituted by protein, rRNA, tRNA and tmRNA coding regions annotated from GenBank prokaryotic genomes (Sayers et al., 2019). Nucleotide sequences from a maximum of 50 different genome assemblies or complete genomes are reported per organism for a total of 70,812 bacterial genomes and 3,007 archaeal genomes. DB2 is the nucleotide database (ftp://ftp.ncbi.nih.gov/blast/db/FASTA/nt.gz) which contains partially non-redundant nucleotide sequences from the GenBank, EMBL and DDBJ databases. The sequence taxonomy of DB1 and DB2 relies on GenBank genome taxonomy (Benson et al., 2018). The two databases can be downloaded from the PhyloPrimer GitHUB page (https://github.com/gvMicroarctic/PhyloPrimer). DB1 and DB2 were last updated contained 261,143,432 (DB1) and 60,553,916 (DB2) entries. The databases cannot be 
373 substituted. However, the user can upload extra sequences for the in silico check; in this case

374 PhyloPrimer will check the taxon-specificity of the oligos against both the DB2 sequences and

375 the user uploaded sequences.

376

377

378

379

380

381

382

383

384

385

386

387

388

389

390

391

392

393

394

395

396

397

398

399

400

401

402

403

404

405

406

407

408

409

410

411

\subsection{PhyloPrimer test}

The primer pairs were designed to amplify all the organisms related to the genus Streptococcus (PP1), and five Streptococcus species: Streptococcus agalactiae (PP2), Streptococcus pneumoniae (PP3), Streptococcus pyogenes (PP4), Streptococcus mutans (PP5) and Streptococcus mitis (PP6) (Table 1). The primer design was performed with PhyloPrimer (Dynamic Selection mode) where six rpoB gene sequences were uploaded for the tree construction (Data S1): one for each Streptococcus species in the mock communities and one sequence belonging to $S$. dysgalactiae which was shown to be highly related to $S$. pyogenes (Jensen \& Kilian, 2012). The primers were designed with default parameters with exception of melting and annealing temperature $\left(60-75^{\circ} \mathrm{C}\right)$, monovalent ion concentration $(0 \mathrm{mM})$, magnesium ion concentration $(2.5 \mathrm{mM})$, oligo concentration $(0.6 \mu \mathrm{M})$ and dNTP concentration $(1.2 \mu \mathrm{M})$ which were modified accordingly to the specifics of the polymerase used for the PCR. Furthermore, in order to be sure the DNA was amplifiable in all the mock communities, the primers $341 \mathrm{~F}$ and $518 \mathrm{R}$ were also used to amplify the $16 \mathrm{~S}$ rRNA gene as a positive control (Table 1) (Muyzer, De Waal \& Uitterlinden, 1993).

The primers were tested with four mock communities: Metagenomic Control Material for Pathogen Detection (ATCC ${ }^{\circledR}$ MSA-4000), 10 Strain Staggered Mix Genomic Material (ATCC ${ }^{\circledR}$ MSA-1001), Skin Microbiome Genomic Mix (ATCC ${ }^{\circledR}$ MSA-1005) and ZymoBIOMICS Microbial Community DNA Standard (D6306, Zymo Research). These communities comprise several organisms, present with different abundances and ranging in microbial diversity. In the following tests they will be called community A, B, C and D, respectively (Table 2). Each mock community DNA was used as template for the PCR amplification using the primers 16S rRNA and the PhyloPrimer developed primer pairs (PP1, PP2, PP3, PP4, PP5 and PP6). The $25 \mu \mathrm{L}$ PCR solution consisted in $12.5 \mu \mathrm{L}$ for $2 \mathrm{X}$ KAPA HiFi HotStart ReadyMix polymerase (KAPA BIOSYSTEMS), $1.5 \mu \mathrm{L}$ of $5 \mu \mathrm{M}$ forward primer, $1.5 \mu \mathrm{L}$ of $5 \mu \mathrm{M}$ reverse primer, between 1-3 $\mu \mathrm{L}$ of template DNA (corresponding to $4 \mathrm{ng}$ of DNA) and nuclease-free water up to volume. A negative control where the template DNA was substituted with nuclease-free water was included for every primer pair.

The PCR was performed using an Eppendorf Mastercycler nexus X2 thermal cycler (Eppendorf) with the following conditions: $95^{\circ} \mathrm{C}$ for 3 minutes, 25 cycles of $98^{\circ} \mathrm{C}$ for 20 seconds, $64^{\circ} \mathrm{C}$ for 15 seconds and $72^{\circ} \mathrm{C}$ for 20 seconds, and a final extension of $72^{\circ} \mathrm{C}$ for 1 minute. The annealing temperature of $64^{\circ} \mathrm{C}$ was used for all the primer pairs PP1, PP2, PP4, PP5 and PP6, whereas we used $65^{\circ} \mathrm{C}$ for $\mathrm{P} 3$ and $62^{\circ} \mathrm{C}$ for the $16 \mathrm{~S}$ rRNA primers. 
412 For each sample, $6 \mu \mathrm{L}$ of PCR product was then run with $2 \mu \mathrm{L}$ of gel loading buffer (NEB) on

$4131.5 \% \mathrm{w} / \mathrm{v}$ horizontal agarose gel $\left(0.5 \mathrm{mg}^{2}\right.$ ethidium bromide $\mathrm{ml}^{-1}$ in $1 \mathrm{x}$ TEA buffer (Tris acetate 414 EDTA) and run for 30 minutes at $120 \mathrm{mV}$ (Bio-Rad PowerPac 300, Bio-Rad Laboratories). Gel 415 pictures were visualized under UV light and captured with GelDoc-ItTS2 Imager (UVP). No 416 bands were shown in any of the negative control lanes. GelPilot 100 bp Plus Ladder (Qiagen) 417 was run for amplicon size comparison.

418

The non-specific amplicon band obtained in community A with the primer pair PP6 was

420 sequenced with the nanopore technology. The library preparation was performed using the SQKLSK109 kit (Oxford Nanopore Technologies, Oxford, UK). The sequencing was performed using a flow cell FLO-MIN106 with a MinION device and can be found in the European Nucleotide Archive (ENA) at EMBL-EBI under accession number PRJEB42474 (https://www.ebi.ac.uk/ena/browser/view/PRJEB42474). The sequences were basecalled using Guppy v 3.2.2 (Oxford Nanopore Technologies). Sequences were then taxonomy assigned by BLAST search against genomes contained in community A. The list of the genomes used to create the BLAST database can be found in Table S1.

\section{8}

429

430

431

432

433

434

435

436

437

438

439

440

441

442

443

444

445

446

447

448

449

\section{Results and discussion}

The development of taxonomic specific primers is essential to many environmental and biomedical biomonitoring and detection studies (e.g. Ai et al., 2019; dos Santos et al., 2020; Liu et al., 2003; Song et al., 2000) where the recent COVID-19 pandemic is a perfect example of how important is the design of species-specific primers to detect a specific organism of interest (Park et al., 2020). We developed PhyloPrimer, an automated platform that integrates a new pipeline which aims to design taxonomic-specific oligos and tests them for secondary structures and target specificity.

The 16S rRNA gene was amplified in all the four communities showing that all the DNA communities had amplifiable microbial DNA (Fig. 5). The primer pair PP1 which was specific for the Streptococcus genus produced amplicons of the expected size (about $500 \mathrm{bp}$ ) in all the communities except from community D where no Streptococcus species were present (Table 2). Primers PP2, PP3 and PP4 which targeted respectively S. agalactiae, S. pneumoniae and S. pyogenes showed PCR products only in community A which was the only community that contained these organisms. The amplicon size also reflected that predicted by PhyloPrimer being around 480, 300 and $400 \mathrm{bp}$ for primer PP2, PP3 and PP4, respectively. The primer pair PP5 only amplified community B which was the only one containing $S$. mutans. Finally, the primer pair PP6, specific for S. mitis, showed bands (around $1000 \mathrm{bp}$ ) in both community A and C. Community A did not contain S. mitis and therefore species specificity was not achieved with this primer pair. This non-specific band is due to the amplification of the other Streptococcus species present in community A (Fig. 5 and Table 2). Of the 268,551 amplified sequences that 
450 matched to genomes present in community A, in fact, more than $99 \%$ of the sequences was

451

452

453

454

455

456

457

458

459

460

461

462

463

464

465

466

467

468

469

470

471

472

473

474

475

476

477

478

479

480

481

482

483

484

485

486

487

488

assigned to the genus Streptococcus. Of these, $66 \%$ of the sequence was assigned to $S$.

pneumoniae, $33 \%$ to $S$. pyogenes, and $1 \%$ to $S$. agalactiae.

Organisms belonging to the species $S$. mitis have been previously observed to not form a wellisolated phylogenetic cluster (Whatmore et al., 2000). In particular, S. mitis has been shown to be closely related to $S$. pneumoniae (Kawamura et al., 1995). The close similarity between these two species can be observed from PhyloPrimer tree where S. mitis and S. pneumoniae organisms do not show well-separated phylogenetic clusters (https://www.cerealsdb.uk.net/cerealgenomics/cgi-bin/tree_paper.cgi). Therefore the nonspecificity of the primer pair PP6 is caused by the scarce differentiation of S. mitis from the other Streptococcus species where, in particular, the non-specific band observed in community A could be due to the amplification of $S$. pneumoniae (Fig. 5). This result is confirmed by the PhyloPrimer in silico taxonomic test where all the designed primer pairs targeting $S$. mitis also targeted $S$. pneumoniae. Furthermore, the positive consensus sequence calculated for $S$. mitis only had a total of 8 differing bases with the negative consensus (Fig. S1); and no differing bases at all between positive and negative consensus within either its forward or reverse primer sequences (i.e. PP6) whereases all the other selected primer pairs (i.e. PP1, PP2, PP3, PP4 and PP5) had differing bases (Fig. S2). It was therefore not possible to design a species specific rpoB primer pair for $S$. mitis as primers could not be made to target any of the eight known bases unique to this species, due to design constraints.

PhyloPrimer performed well with different organism and gene settings and showed overall good results when tested on the mock communities. There are several oligo design software, such as MPrimer (Shen et al., 2010), PrimerDesign-M (Yoon and Leitner, 2015), MRPrimerW (Kim et al., 2016) and Oli2go (Hendling et al., 2018), that are similar to PhyloPrimer at different stages of the pipeline: homolog screening, secondary structure check and oligo scoring. While others implement the use of a positive and negative consensus sequence for oligo design, such as in the case of Morphocatcher (Shirshikov et al., 2019) and Uniqprimer (Karim et al., 2019), PhyloPrimer automizes all the steps, from homologous sequence selection to oligo scoring, providing a user-friendly oligo design platform.

The software also comes with some limitations. For example, the database used for the tree construction contains only microbial sequences, which lack the added complexity of lengthy intron-containing eukaryotic genes. DB1 is also constituted by coding-region sequences and therefore PhyloPrimer cannot build a phylogenetic tree with intergenic regions. Also, PhyloPrimer does not design degenerate oligos specifically. PhyloPrimer uses a consensus approach and it designs the oligos from a consensus sequence calculated from a DNA alignment. Therefore it will not introduce degeneracy on purpose and will design oligos containing

Peer] reviewing PDF | (2020:10:54347:1:1:NEW 8 Feb 2021) 
489

490

491

492

493

494

495

496

497

498

499

500

501

502

503

504

505

506

507

508

509

510

511

512

513

514

515

516

517

518

519

520

521

522

523

524

525

526

degenerate bases only if present in the consensus sequence and if necessary to the design of suitable oligos.

\section{Conclusion}

We developed PhyloPrimer, a semi-automated and user-friendly pipeline to go from sequence selection to design and in silico test oligos. This tool aims to help with oligo design of complex environmental communities speeding up and providing a solid and reproducible pipeline for the oligo design and in silico tests. We showed the relevance of this approach which showed good results in terms of oligo-specificity when tested on microbial mock communities.

\section{Acknowledgements}

We thank Paul Wilkinson for the help given to set up the web server. We thank Ella Boswell and Nina Blampied, from the University of Bristol, and Layla Rahiem and Mirjam Pikaart, from Avans University, who used PhyloPrimer and gave useful feedback in return.

\section{References}

Adékambi T, Drancourt M, Raoult D. 2009. The rpoB gene as a tool for clinical microbiologists. Trends in Microbiology 17:37-45. DOI: 10.1016/j.tim.2008.09.008.

Ai L, Liu J, Jiang Y, Guo W, Wei P, Bai L. 2019. Specific PCR method for detection of species origin in biochemical drugs via primers for the ATPase 8 gene by electrophoresis. Microchimica Acta 186:14-17. DOI: 10.1007/s00604-019-3738-5.

Allawi HT, Santalucia J. 1997. Thermodynamics and NMR of internal G·T mismatches in DNA. Biochemistry 36:10581-10594. DOI: 10.1021/bi962590c.

Allawi HT, SantaLucia J. 1998a. Nearest-neighbor thermodynamics of internal A $\mathrm{C}$ mismatches in DNA: Sequence dependence and pH effects. Biochemistry 37:9435-9444. DOI: 10.1021/bi9803729.

Allawi HT, SantaLucia J. 1998b. Thermodynamics of internal C·T mismatches in DNA. Nucleic Acids Research 26:2694-2701. DOI: 10.1093/nar/26.11.2694.

Allawi HT, SantaLucia J. 1998c. Nearest neighbor thermodynamic parameters for internal G·A mismatches in DNA. Biochemistry 37:2170-2179. DOI: 10.1021/bi9724873.

Baxevanis AD. 2020. Assessing pairwise sequence similarity: BLAST and FASTA. In: Baxevanis AD, Bader GD, Wishart DS eds. Bioinformatics. John Wiley \& Sons, 45-78.

Benson DA, Cavanaugh M, Clark K, Karsch-Mizrachi I, Ostell J, Pruitt KD, Sayers EW. 2018. GenBank. Nucleic Acids Research 46:D41-D47. DOI: 10.1093/nar/gkx1094.

Bommarito S, Peyret N, SantaLucia J. 2000. Thermodynamic parameters for DNA sequences with dangling ends. Nucleic Acids Research. DOI: 10.1093/nar/28.9.1929.

Boyce R, Chilana P, Rose TM. 2009. iCODEHOP: A new interactive program for designing 
527

528

529

530

531

532

533

534

535

536

537

538

539

540

541

542

543

544

545

546

547

548

549

550

551

552

553

554

555

556

557

558

559

560

561

562

563

564

565

566

567

COnsensus-DEgenerate Hybrid Oligonucleotide Primers from multiply aligned protein sequences. Nucleic Acids Research 37:222-228. DOI: 10.1093/nar/gkp379.

Case RJ, Boucher Y, Dahllöf I, Holmström C, Doolittle WF, Kjelleberg S. 2007. Use of 16S rRNA and rpoB genes as molecular markers for microbial ecology studies. Applied and Environmental Microbiology 73:278-288. DOI: 10.1128/AEM.01177-06.

Chuang LY, Cheng YH, Yang CH. 2013. Specific primer design for the polymerase chain reaction. Biotechnology Letters 35:1541-1549. DOI: 10.1007/s10529-013-1249-8.

Deiner K, Bik HM, Mächler E, Seymour M, Lacoursière-Roussel A, Altermatt F, Creer S, Bista I, Lodge DM, de Vere N, Pfrender ME, Bernatchez L. 2017. Environmental DNA metabarcoding: Transforming how we survey animal and plant communities. Molecular Ecology 26:5872-5895. DOI: 10.1111/mec.14350.

Elbrecht V, Hebert PDN, Steinke D. 2018. Slippage of degenerate primers can cause variation in amplicon length. Scientific Reports 8:1-5. DOI: 10.1038/s41598-018-29364-z.

Essinger SD, Reichenberger E, Morrison C, Blackwood CB, Rosen GL. 2015. A toolkit for ARB to integrate custom databases and externally built phylogenies. PLOS ONE 10:1-7. DOI: 10.1371/journal.pone.0109277.

Fierer N, Jackson JA, Vilgalys R, Jackson RB. 2005. Assessment of soil microbial community structure by use of taxon-specific quantitative PCR assays. Applied and Environmental Microbiology 71:4117-4120. DOI: 10.1128/AEM.71.7.4117-4120.2005.

Gaby JC, Buckley DH. 2012. A comprehensive evaluation of PCR primers to amplify the nifH gene of nitrogenase. PLoS ONE 7. DOI: 10.1371/journal.pone.0042149.

Gadberry MD, Malcomber ST, Doust AN, Kellogg EA. 2005. Primaclade - A flexible tool to find conserved PCR primers across multiple species. Bioinformatics 21:1263-1264. DOI: 10.1093/bioinformatics/bti134.

Garibyan L, Avashia N. 2013. Polymerase chain reaction. Journal of Investigative Dermatology 133:1-4. DOI: $10.1038 /$ jid.2013.1.

Goel N, Singh S, Aseri TC. 2013. A comparative analysis of soft computing techniques for gene prediction. Analytical Biochemistry 438:14-21. DOI: 10.1016/j.ab.2013.03.015.

Hendling M, Pabinger S, Peters K, Wolff N, Conzemius R, Barišić I. 2018. Oli2go: An automated multiplex oligonucleotide design tool. Nucleic Acids Research 46:W252-W256. DOI: 10.1093/nar/gky319.

Hugerth LW, Wefer HA, Lundin S, Jakobsson HE, Lindberg M, Rodin S, Engstrand L, Andersson AF. 2014. DegePrime, a program for degenerate primer design for broadtaxonomic-range PCR in microbial ecology studies. Applied and Environmental Microbiology 80:5116-5123. DOI: 10.1128/AEM.01403-14.

Iserte JA, Stephan BI, Goñi SE, Borio CS, Ghiringhelli PD, Lozano ME. 2013. Family-Specific Degenerate Primer Design: A Tool to Design Consensus Degenerated Oligonucleotides. Biotechnology Research International 2013:1-9. DOI: 10.1155/2013/383646.

Jensen A, Kilian M. 2012. Delineation of Streptococcus dysgalactiae, its subspecies, and its clinical and phylogenetic relationship to Streptococcus pyogenes. Journal of Clinical Microbiology. DOI: 10.1128/JCM.05900-11. 
568

569

570

571

572

573

574

575

576

577

578

579

580

581

582

583

584

585

586

587

588

589

590

591

592

593

594

595

596

597

598

599

600

601

602

603

604

605

606

607

608

Johnston AD, Lu J, Ru K lin, Korbie D, Trau M. 2019. PrimerROC: accurate conditionindependent dimer prediction using ROC analysis. Scientific Reports 9:1-14. DOI: 10.1038/s41598-018-36612-9

Kalendar R, Lee D, Schulman A. 2009. FastPCR software for PCR primer and probe design and repeat search. Genes, Genomes and Genomics 3:1-14.

Karim S, Ryan McNally R, Nasaruddin AS, DeReeper A, Mauleon RP, Charkowski AO, Leach JE, Ben-Hur A, Triplett LR. 2019. Development of the automated primer design workflow uniqprimer and diagnostic primers for the broad-host-range plant pathogen dickeya dianthicola. Plant Disease 103:2893-2902. DOI: 10.1094/PDIS-10-18-1819-RE.

Katoh K, Standley DM. 2013. MAFFT multiple sequence alignment software version 7: Improvements in performance and usability. Molecular Biology and Evolution 30:772-780. DOI: $10.1093 / \mathrm{molbev} / \mathrm{mst} 010$.

Kawamura Y, Hou XG, Sultana F, Miura H, Ezaki T. 1995. Determination of 16S rRNA sequences of Streptococcus mitis and Streptococcus gordonii and phylogenetic relationships among members of the genus Streptococcus. International Journal of Systematic Bacteriology 45:406-408. DOI: 10.1099/00207713-45-2-406.

Kent WJ, Sugnet CW, Furey TS, Roskin KM, Pringle TH, Zahler AM, Haussler a. D. 2002. The Human Genome Browser at UCSC. Genome Research 12:996-1006. DOI: 10.1101/gr.229102.

Kim H, Kang NN, An KH, Koo JH, Kim MS. 2016. MRPrimerW: a tool for rapid design of valid high-quality primers for multiple target qPCR experiments. Nucleic acids research 44:W259-W266. DOI: 10.1093/nar/gkw380.

Langmead B. 2010. Aligning short sequencing reads with Bowtie. Current Protocols in Bioinformatics. DOI: 10.1002/0471250953.bi1107s32.

Linhart C, Shamir R. 2005. The degenerate primer design problem: Theory and applications. Journal of Computational Biology. DOI: 10.1089/cmb.2005.12.431.

Liu C, Song Y, McTeague M, Vu AW, Wexler H, Finegold SM. 2003. Rapid identification of the species of the Bacteroides fragilis group by multiplex PCR assays using group- and species-specific primers. FEMS Microbiology Letters 222:9-16. DOI: 10.1016/S03781097(03)00296-9.

Ludwig W, Strunk O, Westram R, Richter L, Meier H, Yadhukumar A, Buchner A, Lai T, Steppi S, Jacob G, Förster W, Brettske I, Gerber S, Ginhart AW, Gross O, Grumann S, Hermann S, Jost R, König A, Liss T, Lüßbmann R, May M, Nonhoff B, Reichel B, Strehlow R, Stamatakis A, Stuckmann N, Vilbig A, Lenke M, Ludwig T, Bode A, Schleifer KH. 2004. ARB: A software environment for sequence data. Nucleic Acids Research 32:1363-1371. DOI: $10.1093 /$ nar/gkh293.

Markham NR, Zuker M. 2008. UNAFold. DOI: 10.1007/978-1-60327-429-6_1.

Morales SE, Holben WE. 2009. Empirical testing of 16S rRNA gene PCR primer pairs reveals variance in target specificity and efficacy not suggested by in silico analysis. Applied and Environmental Microbiology 75:2677-2683. DOI: 10.1128/AEM.02166-08.

Morgulis A, Coulouris G, Raytselis Y, Madden TL, Agarwala R, Schäffer AA. 2008. Database 
609

610

611

612

613

614

615

616

617

618

619

620

621

622

623

624

625

626

627

628

629

630

631

632

633

634

635

636

637

638

639

640

641

642

643

644

645

646

647

648

indexing for production MegaBLAST searches. Bioinformatics 24:1757-1764. DOI:

10.1093/bioinformatics/btn322.

Muyzer G, De Waal EC, Uitterlinden AG. 1993. Profiling of complex microbial populations by denaturing gradient gel electrophoresis analysis of polymerase chain reaction-amplified genes coding for 16S rRNA. Applied and Environmental Microbiology 59:695-700. DOI: 10.1128/aem.59.3.695-700.1993.

Owczarzy R, Moreira BG, You Y, Behlke MA, Wälder JA. 2008. Predicting stability of DNA duplexes in solutions containing magnesium and monovalent cations. Biochemistry 47:5336-5353. DOI: 10.1021/bi702363u.

Owczarzy R, You Y, Moreira BG, Manthey JA, Huang L, Behlke MA, Walder JA. 2004. Effects of Sodium Ions on DNA Duplex Oligomers: Improved Predictions of Melting Temperatures. Biochemistry 43:3537-3554. DOI: 10.1021/bi034621r.

Parada AE, Needham DM, Fuhrman JA. 2016. Every base matters: Assessing small subunit rRNA primers for marine microbiomes with mock communities, time series and global field samples. Environmental Microbiology 18:1403-1414. DOI: 10.1111/1462-2920.13023.

Park M, Won J, Choi BY, Lee CJ. 2020. Optimization of primer sets and detection protocols for SARS-CoV-2 of coronavirus disease 2019 (COVID-19) using PCR and real-time PCR. Experimental \& molecular medicine. DOI: 10.1038/s12276-020-0452-7.

Peyret N, Seneviratne PA, Allawi HT, SantaLucia J. 1999. Nearest-neighbor thermodynamics and NMR of DNA sequences with internal A·A, C.C, G.G, and T·T mismatches. Biochemistry 38:3468-3477. DOI: 10.1021/bi9825091.

Price MN, Dehal PS, Arkin AP. 2009. Fasttree: Computing large minimum evolution trees with profiles instead of a distance matrix. Molecular Biology and Evolution 26:1641-1650. DOI: 10.1093/molbev/msp077.

Rose TM, Henikoff JG, Henikoff S. 2003. CODEHOP (COnsensus-DEgenerate Hybrid Oligonucleotide Primer) PCR primer design. Nucleic Acids Research 31:3763-3766. DOI: 10.1093/nar/gkg524.

Rychlik W. 2007. OLIGO 7 primer analysis software. Methods in Molecular Biology 402:35-59. DOI: $10.1385 / 1-59745-528-8: 35$.

SantaLucia J, Hicks D. 2004. The thermodynamics of DNA structural motifs. Annual Review of Biophysics and Biomolecular Structure 33:415-440. DOI: 10.1146/annurev.biophys.32.110601.141800.

dos Santos EH, Yamamoto L, Domingues W, di Santi SM, Kanunfre KA, Okay TS. 2020. A new Real Time PCR with species-specific primers from Plasmodium malariae/P. brasilianum mitochondrial cytochrome b gene. Parasitology International 76:102069. DOI: 10.1016/j.parint.2020.102069.

Sayers EW, Beck J, Brister JR, Bolton EE, Canese K, Comeau DC, Funk K, Ketter A, Kim S, Kimchi A, Kitts PA, Kuznetsov A, Lathrop S, Lu Z, McGarvey K, Madden TL, Murphy TD, O’Leary N, Phan L, Schneider VA, Thibaud-Nissen F, Trawick BW, Pruitt KD, Ostell J. 2020. Database resources of the National Center for Biotechnology Information. Nucleic 
649

650

651

652

653

654

655

656

657

658

659

660

661

662

663

664

665

666

667

668

669

670

671

672

673

674

675

676

677

678

679

680

681

682

683

684

685

686

687

688

689

690

acids research 48:D9-D16. DOI: 10.1093/nar/gkz899.

Sayers EW, Cavanaugh M, Clark K, Ostell J, Pruitt KD, Karsch-Mizrachi I. 2019. GenBank. Nucleic Acids Research 47:D94-D99. DOI: 10.1093/nar/gky989.

Shafee T, Lowe R. 2018. Eukaryotic and Prokaryotic Gene Structure. SSRN Electronic Journal. DOI: $10.2139 /$ ssrn.3013506.

Shen Z, Qu W, Wang W, Lu Y, Wu Y, Li Z, Hang X, Wang X, Zhao D, Zhang C. 2010. MPprimer: A program for reliable multiplex PCR primer design. BMC Bioinformatics 11. DOI: 10.1186/1471-2105-11-143.

Shirshikov F V., Pekov YA, Miroshnikov KA. 2019. MorphoCatcher: A multiple-alignment based web tool for target selection and designing taxon-specific primers in the loopmediated isothermal amplification method. PeerJ 7. DOI: 10.7717/peerj.6801.

Singh VK, Mangalam AK, Dwivedi S, Naik S. 1998. Primer Premier Program for Design of.pdf. 24:2-3.

Song YL, Kato N, Liu CX, Matsumiya Y, Kato H, Watanabe K. 2000. Rapid identification of 11 human intestinal Lactobacillus species by multiplex PCR assays using group- and speciesspecific primers derived from the 16S-23S rRNA intergenic spacer region and its flanking 23S rRNA. FEMS Microbiology Letters 187:167-173. DOI: 10.1016/S03781097(00)00196-8.

Sorek R, Cossart P. 2010. Prokaryotic transcriptomics: A new view on regulation, physiology and pathogenicity. Nature Reviews Genetics 11:9-16. DOI: 10.1038/nrg2695.

Takahashi S, Tomita J, Nishioka K, Hisada T, Nishijima M. 2014. Development of a prokaryotic universal primer for simultaneous analysis of Bacteria and Archaea using next-generation sequencing. PLoS ONE 9. DOI: 10.1371/journal.pone.0105592.

Untergasser A, Cutcutache I, Koressaar T, Ye J, Faircloth BC, Remm M, Rozen SG. 2012. Primer3-new capabilities and interfaces. Nucleic Acids Research 40:1-12. DOI: 10.1093/nar/gks596.

Untergasser A, Nijveen H, Rao X, Bisseling T, Geurts R, Leunissen JAM. 2007. Primer3Plus, an enhanced web interface to Primer3. Nucleic Acids Research 35:71-74. DOI: 10.1093/nar/gkm306.

Untergasser A, Cutcutache I, Koressaar T, Ye J, Faircloth BC, Remm M, Rozen SG. 2012. Primer3-new capabilities and interfaces. Nucleic Acids Research 40:1-12. DOI: $10.1093 / \mathrm{nar} / \mathrm{gks} 596$.

Wang S, Liu Y, Liu G, Huang Y, Zhou Y. 2017. A New Primer to Amplify pmoA Gene From NC10 Bacteria in the Sediments of Dongchang Lake and Dongping Lake. Current Microbiology 74:908-914. DOI: 10.1007/s00284-017-1260-8.

Whatmore AM, Efstratiou A, Pickerill AP, Broughton K, Woodard G, Sturgeon D, George R, Dowson CG. 2000. Genetic relationships between clinical isolates of Streptococcus pneumoniae, Streptococcus oralis, and Streptococcus mitis: Characterization of "atypical" pneumococci and organisms allied to S. mitis harboring S. pneumoniae virulence factorencoding genes. Infection and Immunity 68:1374-1382. DOI: 10.1128/IAI.68.3.13741382.2000 .

Yoon H, Leitner T. 2015. PrimerDesign-M: A multiple-alignment based multiple-primer design 
691 tool for walking across variable genomes. Bioinformatics 31:1472-1474. DOI:

692 10.1093/bioinformatics/btu832.

693 You I, Kim EB. 2020. Genome-based species-specific primers for rapid identification of six

694 species of Lactobacillus acidophilus group using multiplex PCR. PLoS ONE 15:1-9. DOI:

$695 \quad$ 10.1371/journal.pone.0230550.

$696 \mathrm{Yu}$ Y, Lee C, Kim J, Hwang S. 2005. Group-specific primer and probe sets to detect

697 methanogenic communities using quantitative real-time polymerase chain reaction.

698 Biotechnology and Bioengineering 89:670-679. DOI: 10.1002/bit.20347.

699 Yu B, Zhang C. 2011. In silico PCR analysis. Methods in Molecular Biology. DOI: 10.1007/978-

700 1-61779-176-5_6.

701 
Figure 1

PhyloPrimer structure indicating the web pages and the server-side processes. 


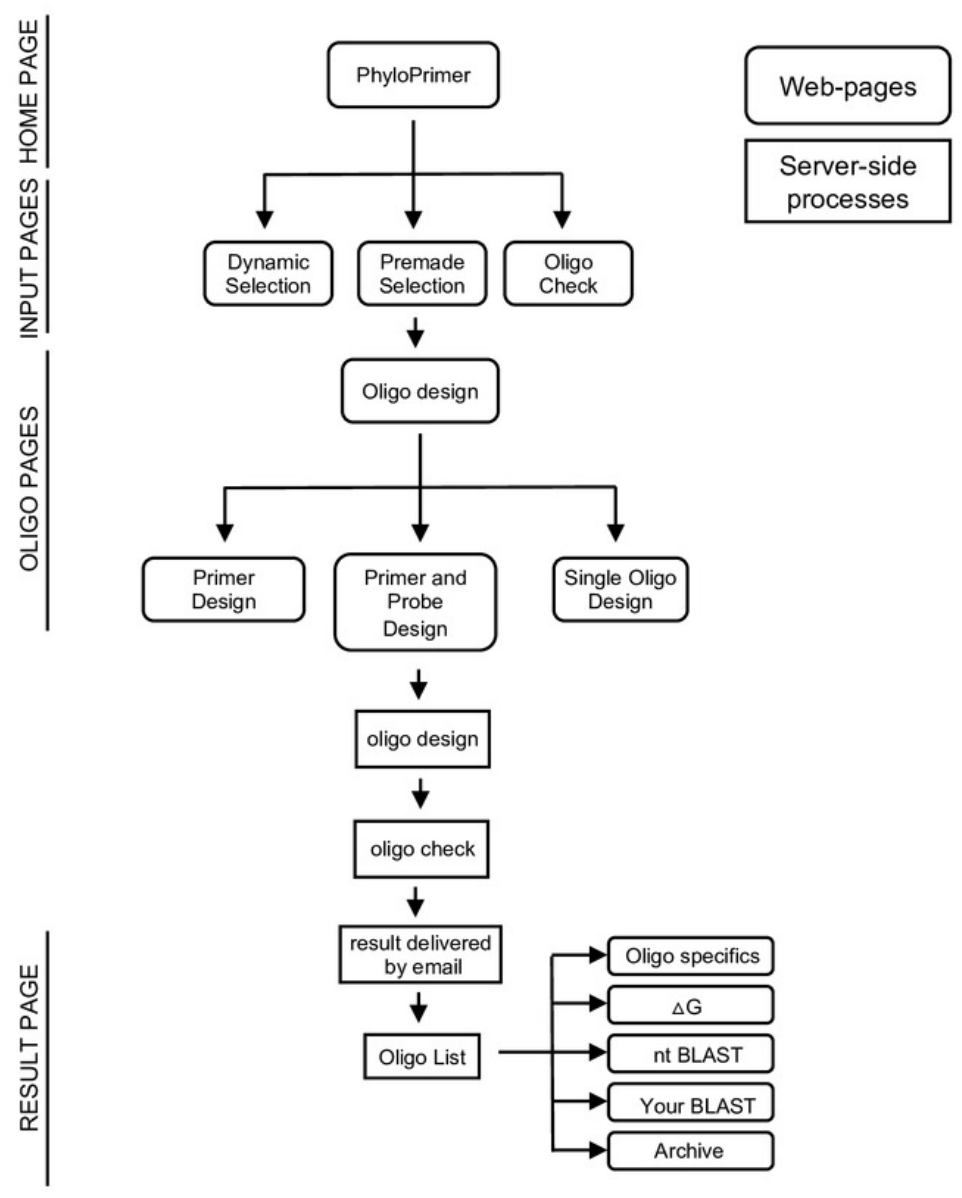




\section{Figure 2}

Detailed scheme of the three different input pages and workflows.

The three workflows are Dynamic Selection (A), Premade Selection (B) and Oligo Check (C). Through the Dynamics Selection page the user can input three different kind of data: up to 10 genes or DNA regions of interest, up to 500 DNA sequences and a Newick tree (together with an alignment file). The Premade Selection page permits the uploading of up to 1500 DNA sequences, 1500 DNA aligned sequences or directly the consensus sequence that will be used for the oligo design. In the Oligo Check page only the upload of predesigned oligos is allowed. Different processes on the server-side of PhyloPrimer will start in relation to which data was uploaded. *There can be optional input files for taxonomy and protein information. If a Newick file is the input, an additional alignment file must be uploaded. ** Oligos are intended as primers pairs, primer pairs plus a probe or single oligos. 


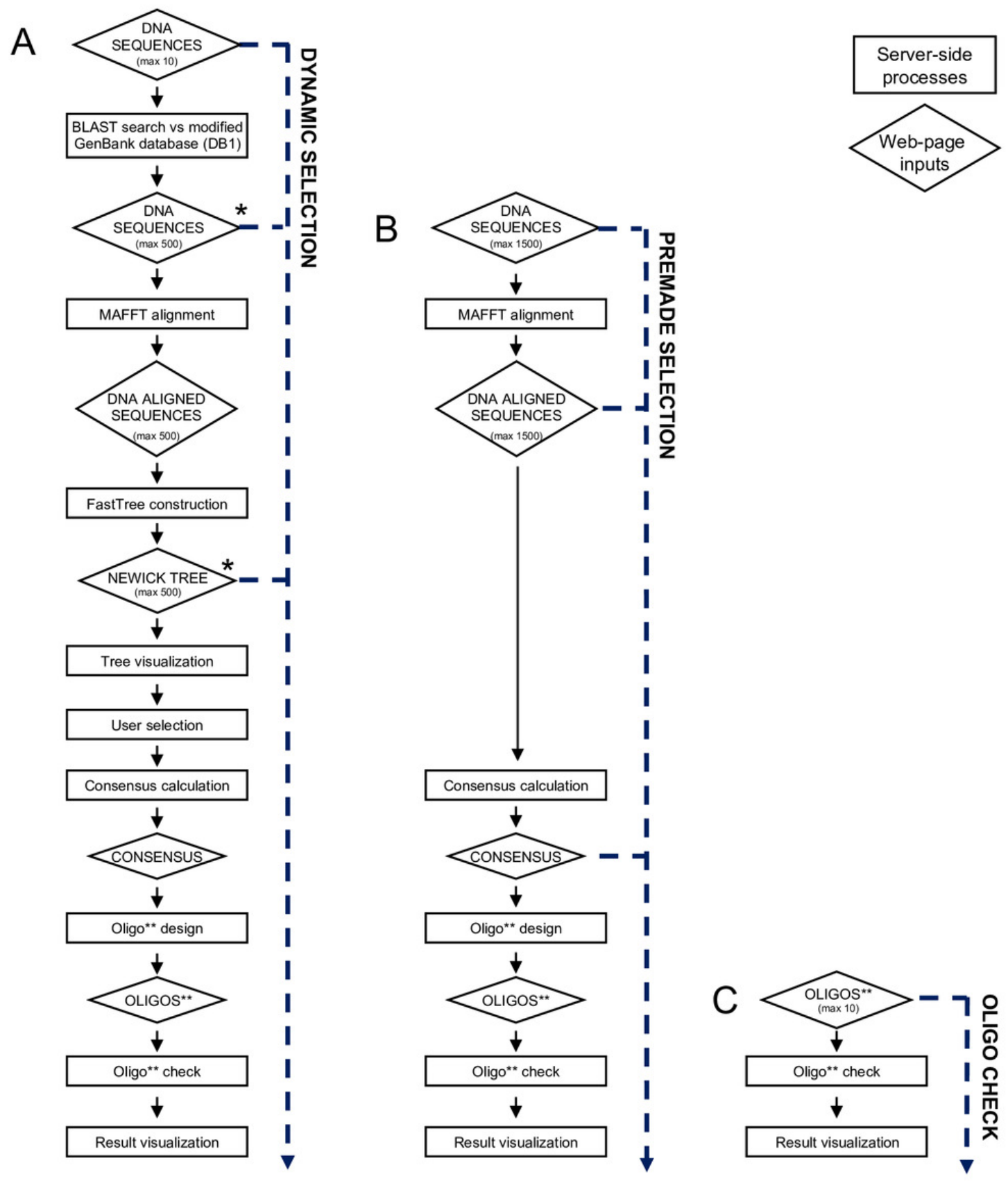




\section{Figure 3}

PhyloPrimer positive and negative consensus workflow.

In the Dynamic Selection mode, the consensus design starts with the selection of DNA sequences on the phylogenetic tree (A). Successively, the selected sequences (i.e. lacZ_1, lacZ_2 and lacZ_3) are used to construct the positive consensus and the others are used to calculate the negative consensus (i.e. lacZ_4, lacZ_5 and lacZ_6). The two consensus sequences are compared, positive consensus areas with differing bases are identified and oligos specific to that area are selected (B). In any consensus visualization of the consensus sequence PhyloPrimer is then going to report only the positive consensus with marked letters where differing with the negative sequence (C). The base color code is as follows: red letters indicate positions where the two sequences presented differing bases, blue letters indicate positions where there are bases on the positive consensus but gaps in the negative and bold letters flank regions where there were gaps on the positive consensus but bases on the negative consensus. A degenerate base is marked as differing only if that base does not contain the corresponding base of the negative consensus. 
A

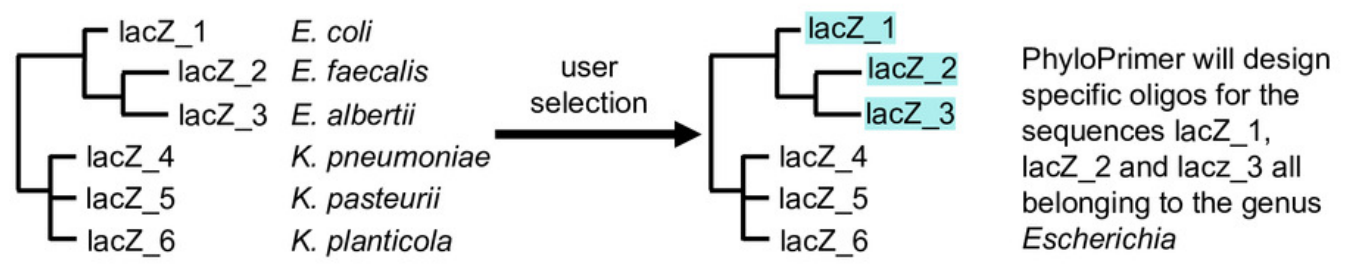

B

Target sequences: lacZ_1, lacZ_2 and lacZ_3 Target sequences: all excluded lacZ_1, lacZ_2 and lacZ_3

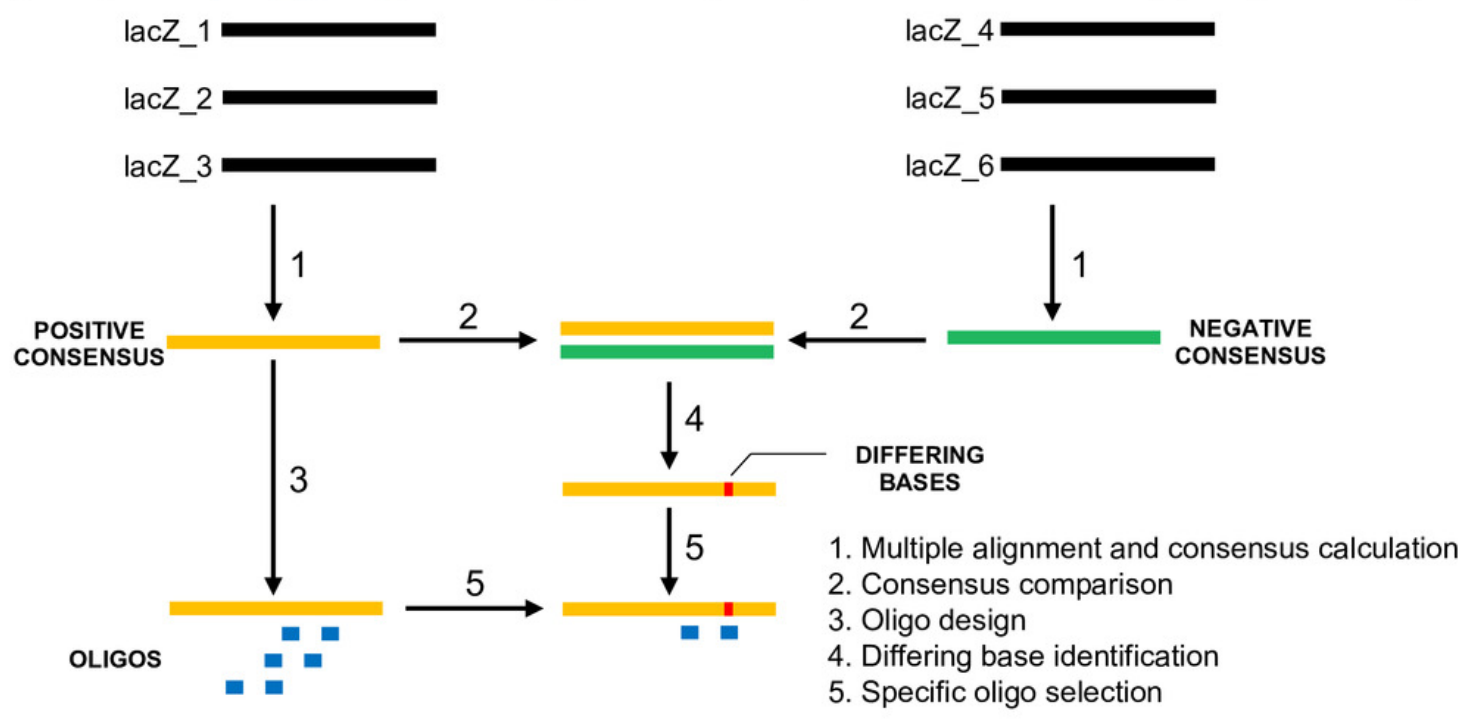

C

POSITIVE CONSENSUS ARGCWGGAAVYCCATGTAAAAAATGTGGARRWGARCTWAAAAAAATAGAA NEGATIVE CONSENSUS ATGMRGGARRAMMMTGY---VWVTGYGGWGRRRHWYTHRMRBCMRWWSAH

POSITIVE CONSENSUS GTDGATTTTTGGAAT---GAYAAAAAATACAC------CTAYAA

NEGATIVE CONSENSUS RYHGAYTTYKKRDMKSYRGRWAKAWWMGADVMKSTSARRSSHRM

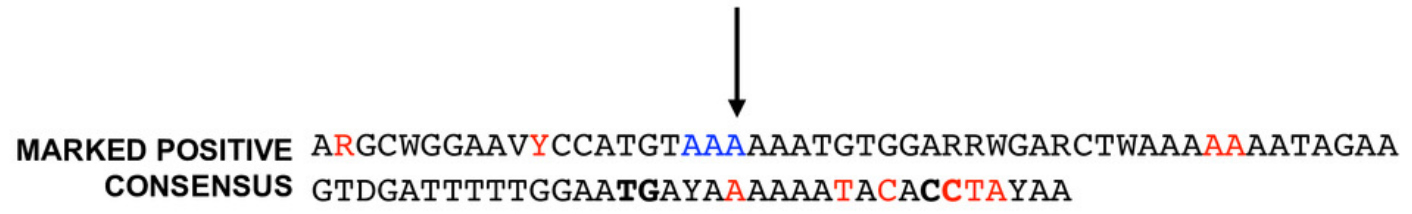




\section{Figure 4}

Primer design workflow.

Oligo design, check and scoring processes are indicated. $* 250$ if no negative consensus was present, no differing bases between the two consensus sequences were present or no differences were taken in consideration in the scoring system. ${ }^{* *}$ depending on the visualization criteria that were selected, +20 and -40 points are assigned if the different oligos BLAST searched against DB2 entries belonged to genera, families, orders, classes, phyla and domains that were selected from the phylogenetic tree. 


\section{Processes oligo design oligo check oligo scoring}

\begin{tabular}{|c|c|}
\hline A & For each possible oligo length, the consensus is chopped into $n$ oligos with $a+1$ shift \\
\hline B & $\begin{array}{l}\text { All the oligos are checked for: } \\
\text { - Homopolymers } \\
\text { - Dinucleotide repetitions } \\
\text { - Degeneracy } \\
\text { - GC\% } \\
\text { - Uniqueness in the consensus }\end{array}$ \\
\hline C & Forward primer = oligo $\quad$ All the oligos are converted into their reverse complement \\
\hline D & $\begin{array}{ll}\text { All the forward primers are checked for: } & \text { All the reverse primer are checked for: } \\
\text { - } T_{m} & \text { - } T_{m} \\
\text { - Degenerate bases in tails } & \text { - Degenerate bases in tails } \\
\text { - GC clamp } & \text { - GC clamp } \\
\text { - Self-dimer } \Delta G & \text { - Self-dimer } \Delta G \\
\text { - Hairpin } \Delta G & \text { - Hairpin } \Delta G\end{array}$ \\
\hline $\mathbf{E}$ & Create primer pairs \\
\hline $\mathbf{F}$ & $\begin{array}{l}\text { All the primer pairs are checked for: } \\
\text { - } T_{a} \\
\text { - } T_{m} \text { difference between forward and reverse primers }\end{array}$ \\
\hline G & $\begin{array}{l}\text { PhyloPrimer assign points to each primer pair following this criteria: } \\
\text { - Differing bases in the second to last base at the } 3 \text { ' end: }+10 \text {. } \\
\text { - Differing bases in the last base at the } 3^{\prime} \text { end: }+20 \text {. } \\
\text { - Differing bases in the rest of the oligo sequence: }+2 \text {. } \\
\text { - Tm difference between forward and reverse primers is lower than } 1:+1 \text {. } \\
\text { - The } \Delta \mathrm{G} \text { is higher than } 1 \mathrm{kcal}^{-1}:+1 \text { (for hairpins and self-dimers). } \\
\text { - Degenerate bases: }-2 \text { if } \mathrm{R}, \mathrm{Y}, \mathrm{S}, \mathrm{W}, \mathrm{K} \text { and } \mathrm{M},-3 \text { if } \mathrm{B}, \mathrm{D}, \mathrm{H} \text { and } \mathrm{V} \text { and }-4 \mathrm{if} \mathrm{N} \text {. }\end{array}$ \\
\hline $\mathbf{H}$ & The first best $1000^{*}$ primer pairs are checked for cross-dimer formation. \\
\hline $\mathbf{I}$ & $\begin{array}{c}\text { The first best } 500^{*} \text { primer pairs are checked with a BLAST and Bowtie search against the } \\
\text { database DB2. }\end{array}$ \\
\hline $\mathbf{J}$ & $\begin{array}{l}\text { The BLAST results are retrieved and the points are assigned to each primer pair as follows: } \\
\text { - Species that was selected in the dynamic tree: }+5 \text {. } \\
\text { - Species** that was selected in the dynamic tree and has been found for the first } \\
\text { time: }+20 \text {. } \\
\text { - Species** which was not selected in dynamic tree: }-40 \text {. }\end{array}$ \\
\hline $\mathbf{L}$ & The first 100 primer pairs are retrieved and visualized in the result page \\
\hline
\end{tabular}


Figure 5

Agarose gel pictures of the PCR products amplified with the 16S rRNA and the PhyloPrimer designed primer (PP1, PP2, PP3, PP4, PP5 and PP6) on the mock communities $A, B, C$ and $D$.

The white star marks the non-specific band found in community A for the primer PP6. All the other primer pairs amplified only the expected communities and no false negatives occurred. 


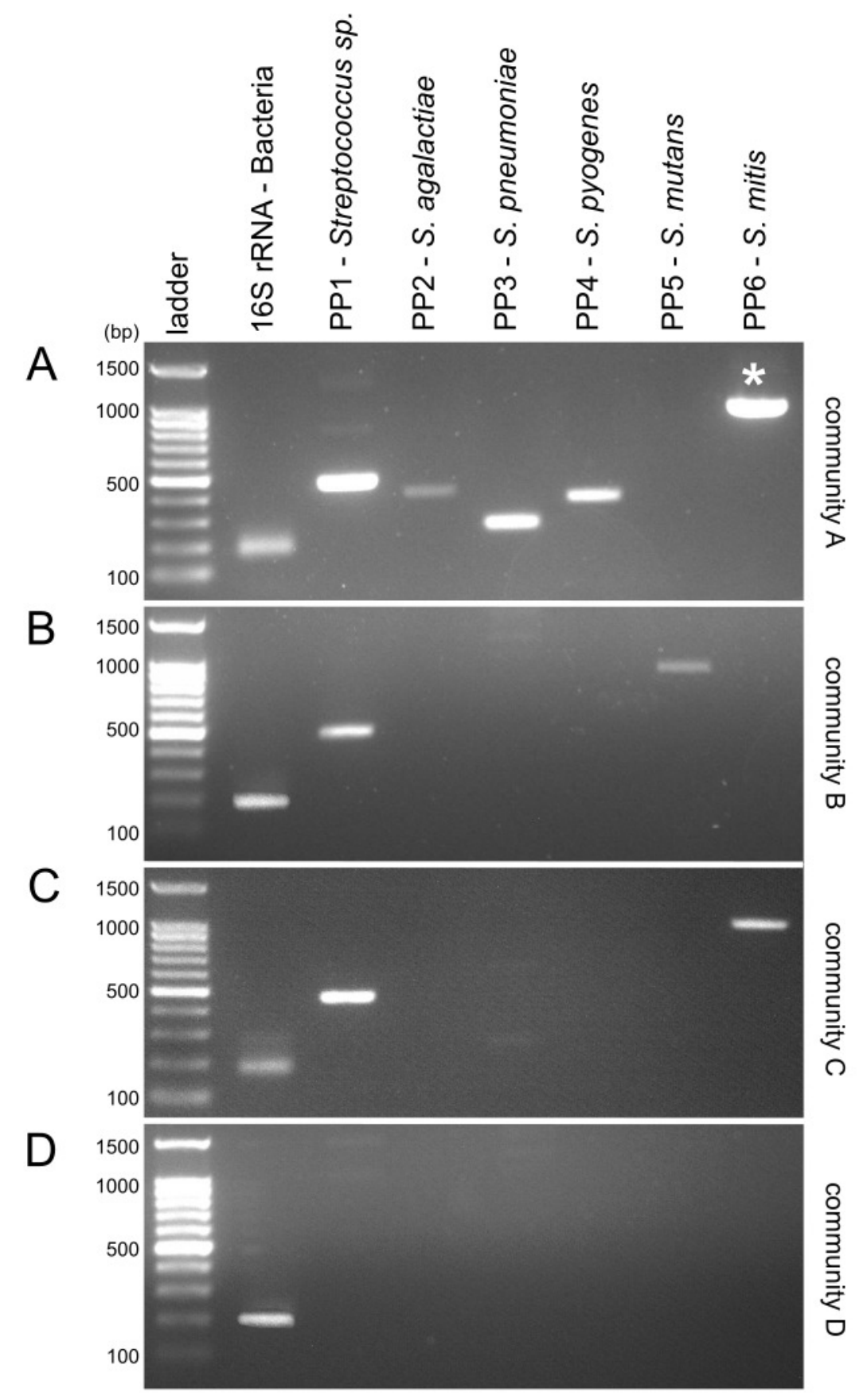




\section{Table 1 (on next page)}

\section{Primer specifics.}

All the primers were designed with PhyloPrimer web platform except the 16S rRNA primers which were designed by Muyzer et al., 1993. *PhyloPrimer predicted amplicon length. 


\begin{tabular}{ccc}
\hline \multirow{2}{*}{ Primers } & \multicolumn{2}{c}{ Primer sequences } \\
\cline { 2 - 3 } 16S rRNA & CCTACGGGAGGCAGCAG & GGCACAGCCTGACGTTGCAT \\
PP1 & TTGACWCGTGACCGTGCTGG & GGCACAGCCTGACGTTGCAT \\
PP2 & GCGTCGCGAAGATGGTTCT & ACCTCAGCACCAATGCGGATGA \\
PP3 & AGCTTGCTTGTRGCTCGCTT & CTCAGTCACAACGGCTGCATCG \\
PP4 & CAGTTGCACAGGCCAATTCGA & GTGAGCCATCTTGACGACGGAT \\
PP5 & GCGAGCGTCTTGTCAAGGAT & ACCACCAAGCGGCTGTTGA \\
PP6 & ACATGCAACGTCAGGCTGT & AGTACGAGCAGCCATACCAAGG \\
\hline Primers & Target organisms & Amplicon size (bp)* \\
\hline 16S rRNA & Bacteria & 200 \\
PP1 & Streptococcus sp. & 470 \\
PP2 & Streptococcus agalactiae & 410 \\
PP3 & Streptococcus pneumoniae & 870 \\
PP4 & Streptococcus pyogenes & 1000 \\
PP5 & Streptococcus mutans & 380 \\
PP6 & Streptococcus mitis & 270 \\
\hline
\end{tabular}

2

3

4

5

6

12

13 


\section{Table 2 (on next page)}

Mock microbial composition.

Composition for the communities A, B, C and D where community A corresponds to ATCC ${ }^{\circledast}$ MSA-4000, B to ATCC ${ }^{\circledR}$ MSA-1001, C to ATCC ${ }^{\circledast}$ MSA-1005 and D to the ZymoBIOMICS community. 
1

\begin{tabular}{|c|c|c|c|c|}
\hline \multirow{2}{*}{ Species } & \multicolumn{4}{|c|}{ Relative abundance (\%) } \\
\hline & $\mathbf{A}$ & $\mathbf{B}$ & $\mathbf{C}$ & D \\
\hline Acinetobacter baumannii & 0.1 & - & - & - \\
\hline Acinetobacter johnsonii & - & - & 16.7 & - \\
\hline Bacillus cereus & - & 4.48 & - & - \\
\hline Bacillus subtilis & - & - & - & 12 \\
\hline Bifidobacterium adolescentis & - & 0.04 & - & - \\
\hline Clostridium beijerinckii & - & 0.45 & - & - \\
\hline Corynebacterium striatum & - & - & 16.7 & - \\
\hline Cryptococcus neoformans & - & - & - & 12 \\
\hline Cutibacterium acnes & - & - & 16.7 & - \\
\hline Deinococcus radiodurans & - & 0.04 & - & - \\
\hline Enterococcus faecalis & 0.7 & 0.04 & - & 12 \\
\hline Escherichia coli & 1.4 & 4.48 & - & 12 \\
\hline Klebsiella pneumoniae & 14.4 & - & - & - \\
\hline Lactobacillus fermentum & - & - & - & 12 \\
\hline Lactobacillus gasseri & - & 0.45 & - & - \\
\hline Listeria monocytogenes & - & - & - & 12 \\
\hline Micrococcus luteus & - & - & 16.7 & - \\
\hline Neisseria meningitidis & 28.9 & - & - & - \\
\hline Pseudomonas aeruginosa & 0.3 & - & - & 12 \\
\hline Rhodobacter sphaeroides & - & 44.78 & - & - \\
\hline Saccharomyces cerevisiae & - & - & - & 12 \\
\hline Salmonella enterica & - & - & - & 12 \\
\hline Staphylococcus aureus & 15.1 & - & - & 12 \\
\hline Staphylococcus epidermidis & - & 44.78 & 16.7 & - \\
\hline Streptococcus agalactiae & 2.9 & - & - & - \\
\hline Streptococcus mitis & - & - & 16.7 & - \\
\hline Streptococcus mutans & - & 0.45 & - & - \\
\hline Streptococcus pneumoniae & 28.9 & - & - & - \\
\hline Streptococcus pyogenes & 7.2 & - & - & - \\
\hline
\end{tabular}

2 